\section{Epidemiology of Urticaria Chronic in Kinshasa}

\author{
Muteba Baseke $^{1^{*}}$ and Ramata Boye ${ }^{2}$ \\ 'Service de Dermatologie, Hôpital General de Référence de Matete, \\ Kinshasa, Congo
}

${ }^{2}$ Service de Dermatologie, Centres Hospitaliers Akram, Kinshasa, Congo

\begin{abstract}
Urticaria is a common dermatologic disorder in both children and adults, affecting $15-20 \%$ of the world's population. When it lasts more than 6 weeks, it is called chronic urticaria whose prevalence varies between 0.1 and $3 \%$. It is a cosmopolitan pathology whose etiology is unknown. Chronic urticaria has a significant negative impact on the quality of life of those who suffer from it.

It is a retrospective and multicenter study over a period of six months, from May 09, 2016 to October 8, 2016. It concerned patients who consulted for ambulatory chronic urticaria in the following hospitals in Kinshasa: Hôpital du Cinquantenaire de Kinshasa/ Kasa-vubu, Clinique Bondeko/Limete, Centres Hospitaliers Akram/ Gombe et Hôpital General de référence de Matete/Matete. 135 patients were the definitive sample of this study.

1525 patients were received for dermatoses including 220 for urticaria. 135 had chronic urticaria, i.e., $8.8 \%$ of dermatoses and $61.3 \%$ of urticaria. $66 \%$ of chronic urticaria were spontaneous and $34 \%$ physical. The female sex was in the majority by contribution to the male sex. The oldest patient was 3 years old and the oldest 88 years old. The mean age of participants was 30.5 years, the age group 31-45 years were the most affected chronic urticaria is a frequent affliction in the middle because reaching $8.8 \%$ of dermatoses with a female predominance.
\end{abstract}

\section{Introduction}

Urticaria is one of the most common dermatological disorders in both children and adults, as $15-20 \%$ of the population has had or will have at least one urticaria and / or angio-Edema during its lifetime. When it lasts more than 6 weeks, chronic urticaria prevalence ranges from 0.1 to $3 \%[1,2]$, women are more commonly affected than men (3.8 times) [2], It is a frequent pathology whose etiology is unknown [3], although in $45 \%$ of cases an autoimmune etiology is called into question [4]. Chronic urticaria has a significant negative impact on quality of life and patients with chronic urticaria have poorer quality of life than those suffering from allergic respiratory diseases or

*Corresponding author: Muteba Baseke, Service de Dermatologie, Hôpital General de Référence de Matete, Kinshasa, Congo, Tel: +243 811865445; E-mail: cbaseke@yahoo.fr

Citation: Baseke M, Boye R (2017) Epidemiology of Urticaria Chronic in Kinshasa. J Clin Dermatol Ther 4: 026.

Received: March 15, 2017; Accepted: September 11, 2017; Published: September 26, 2017 psoriasis [5]. Clinically, it manifests itself as a fleeting eruption made of erythematous plaques, papular "ortié", pruriginous, persisting from a few minutes to a few hours, disappearing without leaving traces, and migrating, other elements appearing elsewhere.

The lesion of urticaria corresponds to an edema which may be dermal or dermo-hypodermic due to phenomena of vasodilation with increased capillary permeability following an influx of inflammatory mediators whose main is histamine and also prostaglandins, leukotrienes, cytokines, etc. These mediators have cellular, plasma, nervous and alimentary origin. The activation of mast cells is the cause of urticaria. Immunological or non-immunological mechanisms may be responsible for this activation [6]. In the Democratic Republic of the Congo, in Kinshasa, we have not found in the literature data on the state of this cosmopolitan pathology. Thus, the aim of this study is to determine the epidemiological and clinical profile of chronic urticaria in order to improve therapeutic and psychosocial management.

\section{Methodology}

This was a retrospective, multicenter study that took place over a six-month period from May 09, 2016 to October 8, 2016. It involved patients who had been referred for ambulatory chronic urticaria in four dermatology departments of Kinshasa: Hôpital du Cinquantenaire de Kinshasa/Kasa-vubu ( $\mathrm{n}=23)$, Clinique Bondeko/Limete $(n=37)$, Centres Hospitaliers Akram/Gombe $(n=45)$, Hôpital General de référence de Matete/Matete $(\mathrm{n}=30)$. A total of 135 patients were the final sample of this study. The variables studied were: age, sex, occupation, marital status, history of allergic and / or autoimmune diseases, diagnosis, paraclinic assessment and treatment.

We obtained the informed consent of all the participants and all the information gathered in the selected files remained confidential, the security measures concerning confidentiality were guaranteed: anonymity, limited access to data, location, the inability to identify subjects when publishing the study results. The data was entered on Epi data. After checking, these were exported to SPSS 15.0. The results are expressed in absolute frequency and in proportions or percentage. The threshold value of statistical significance was at $\mathrm{p}<0.05$.

\section{Results}

Out of 1525 outpatient dermatology patients in selected hospitals, only 220 were suffering from urticaria, including 135 chronic urticaria (all etiology combined), a frequency of $8.8 \%$ of dermatoses and $61.3 \%$ of urticaria.

$66 \%$ of chronic urticaria were spontaneous and $34 \%$ physical (Table 1).

\begin{tabular}{|c|c|}
\hline Types & $\%$ \\
\hline Chronic urticaria physical & 34 \\
\hline Chronic urticaria spontaneous & 66 \\
\hline
\end{tabular}

Table 1: Types of chronic urticaria. 
The female sex was the majority, 85 patients compared to 50 male (Table 2).

\begin{tabular}{|c|c|}
\hline Sex & $\%$ \\
\hline Male & 37 \\
\hline Female & 63 \\
\hline \multicolumn{2}{|c|}{ Table 2: Distribution by sex. } \\
\hline
\end{tabular}

The patient's age ranged from 3 to 88 years. The mean age of the participants was 30.5 years, patients between the ages of $31-45$ years were the most affected.

Clinically, we have pointed out the oedematous papuleuses in $25 \%$ of the cases, lesions of scratching in $37 \%$ of the cases and the positive dermographism in $12 \%$ of the cases (Table 3 ).

\begin{tabular}{|c|c|}
\hline Clinical Lesions of Urticaria & $\%$ \\
\hline Oedematous papules & 25 \\
\hline Lesions of scratching & 37 \\
\hline dermographisme & 12 \\
\hline No lesion & 26 \\
\hline
\end{tabular}

Table 3: Types of clinical lesions.

Almost half of the patients had already had a hives crisis in the past. The majority of patients (95) reported that their quality of life was severely affected during crises. 68 patients, that is to say $50 \%$, are said to practice self-medication.

Acne (25\%) and mycoses (20\%) were the most common dermatoses associated, sinusitis and asthma were the non-dermatological pathologies encountered in 32 and 15 patients, respectively.

$38 \%$ of the patients were employees and $32 \%$ of the students. In the majority of cases, in 81 patients, we were unable to identify the probable cause of chronic urticaria.

The inflammatory assessment performed in 80 patients revealed an increased VS in $5 \%$ of cases, leucocytosis in $3 \%$ of the cases. FAN (Anti-Nuclear Factor) had returned positive in $1 \%$ of cases, thyroid function was disrupted in 7 patients. $3 \%$ of patients were diabetic. The majority of patients were treated with antihistamines: anti-H2 alone $(68 \%)$, in combination with anti-H1 $(30 \%)$, in $2 \%$ of the cases a corticosteroid was necessary to stabilize the patient.

\section{Comments}

In our series, chronic urticaria accounted for $8.8 \%$ of dermatoses, this frequency differs according to study environments. Balp et al., in a study in $5 \mathrm{EU}$ countries (France, Germany, Italy, Spain and the United Kingdom) had a frequency of $0.5 \%$ and $0.2 \%$ [7]. The spontaneous chronic urticaria were the most encountered compared to the chronic physical urticaria, this observation follows that of Zhong et al. [8]. A strong female predominance was noted in relation to the male sex, Gaig et al., in Spain [9], Zuberbier et al., in Germany [10], Zhong in China [10]. This could be explained by the fact that a woman would be more sensitive than the man to the different nociceptive stimuli so they consult more than men.

An average age of 30.5 years was found with age extremes of 3-88 years, in the literature variable data are found, Zhong in China [8] had an average age of 34.7 years, Zaraa in Tunisia [11] had 36 years with the extremes of age of 1-79 years, data superior to ours.
More than half of the patients stated that this condition affected their quality of life, this is in line with the studies by Balp et al., [7] and Zhong and $\mathrm{Col}$ [8]. This is due to the fact that the resulting pruritus makes this pathology invalid and difficult to live. In the majority of cases chronic urticaria was idiopathic, similar data are reported in the literature [11-13]. Idiopathic urticaria was retained as a diagnosis of elimination when all known causes accessible to a reasonable balance sheet were discarded. This is a waiting frame since it reflects the insufficient knowledge of the factors that can trigger hives.

The paraclinic results revealed positive FAN in $1 \%$ of cases, thyroid function disrupted in 5 patients, increased VS in $2 \%$ of patients; These findings are consistent with the relationship between chronic urticaria and autoimmune diseases, as cited in the literature and some studies on this subject $[11,14]$. This can be justified by a high prevalence of autoantibodies and the existence of a chronic inflammatory process during these diseases.

Second-generation antihistamines were prescribed for first-line treatment in all patients, $2 \%$ of whom had oral corticosteroids due to the persistence of pruritus despite the use of antihistamines. 2nd generation antihistamines are the treatment of choice in first intention and when the cause is identified it must of course be taken into account. Our patients showed a significant improvement following regular treatment with antihistamines. We have not found in the literature elements in favor of a given molecule $[15,16]$.

\section{Conclusion}

Chronic urticaria is a frequent affliction in hospitals because reaching $8.8 \%$ of dermatoses, women are more affected than men. It predominantly reaches the active adult population and the psycho-social repercussions are not negligible.

\section{References}

1. Nosbaum A, Augey F, Nicolas JF, Bérard F (2010) Physiopathologie de l'urticaire et approches thérapeutiques. Rev Med 31: 18-22.

2. Gaig P, Olona M, Munoz Lejarazu D, Caballero MT, Domínguez FJ, et al. (2004) Epidemiology of urticaria in Spain. J Investig Allergol Clin Immunol 14: 214-220.

3. Kaplan AP (2012) Treatment of chronic spontaneous urticaria. Allergy Asthma Immunol Res 4: 326-331.

4. Kaplan AP, Greaves M (2009) Pathogenesis of chronic urticaria. Clin Exp Allergy 39: 777-787.

5. Grob JJ, Revuz J, Ortonne JP, Auquier P, Lorette G, et al. (2005) Comparative study of the impact of chronic urticaria, psoriasis and atopic dermatitis on the quality of life. Br J Dermatol 152: 289-295.

6. http://www.medecine.ups-tlse.fr/DCEM2/module8/item114/textel3.htm.

7. Balp MM, Vietri J, Tian H, Isherwood G (2015) The Impact of Chronic Urticaria from the Patient's Perspective: A Survey in Five European Countries. Patient 8: 551-558.

8. Zhong H, Song Z, Chen W Li H, He L, et al. (2014) Chronic urticaria in Chinese population: a hospital-based multicenter epidemiological study. Allergy 69: 359-364.

9. Gaig P, Olona M, Muñoz Lejarazu D, Caballero MT, Domínguez FJ, et al. (2004) Epidemiology of urticaria in Spain. J Investig Allergol Clin Immunol 14: 214-220.

10. Zuberbier T, Balke M, Worm M, Edenharter G, Maurer M (2010) Epidemiology of urticaria: a representative cross-sectional population survey. Clin Exp Dermatol 35: 869-873. 
11. Zaraa I, Sliti N, Ben Kheder Z, El Euch D, Trojjet S, et al. (2010) [Chronic urticaria: a report of 233 cases]. Tunis Med 88: 794-798.

12. Conférence de consensus. Texte long. Prise en charge de l'urticaire chronique (2003) Ann Dermatol Venereol 130: 182-192.

13. Aractingi S (2004) Formes cliniques de l'urticaire chez l'adulte. Revue Française d'Allergologie et d'Immunologie Clinique 44: 103-107.

14. Confino-Cohen R, Chodick G, Shalev V, Leshno M, Kimhi O, et al. (2012) Chronic urticaria and autoimmunity: associations found in a large population study. J Allergy Clin Immunol 129: 1307-1313.
15. Vigan M (2004) Place des antihistaminiques dans la prise en charge des urticaires. Revue Française d'Allergologie et d'Immunologie Clinique 44: $120-124$.

16. Juregui I, Ferrer M, Montoro J, Dávila I, Bartra J, et al. (2007) Antihistamines in the treatment of chronic urticaria. J Investig Allergol Clin Immunol 17: 41-52. 